\title{
Développement de la petite pisciculture marchande au Cameroun : la recherche-action en partenariat
}

Olivier Mikolasek ${ }^{1}$

Blandine Barlet ${ }^{2}$

Eduardo $\mathrm{Chia}^{2}$

Victor Pouomogne 3

Minette Tomedi Eyango Tabi ${ }^{4}$

1 Cirad,

Département Persyst

UPR aquaculture et gestion

des ressources aquatiques

Cemagref - TA B-20/01

34033 Montpellier cedex 01

France

<olivier.mikolasek@cirad.fr>

2 Cirad,

UMR innovation

Département ES du Cirad

Montpellier

France

<blandinebarlet@hotmail.com>

<eduardo.chia@cirad.fr>

${ }^{3}$ Irad

Station de recherche de Foumban

Foumban

Cameroun

<pouomognev@yahoo.fr>

${ }^{4}$ Département de foresterie

Faculté d'agronomie et des sciences agricoles Dschang

Cameroun

<tomedi_tabi@yahoo.fr>

\begin{abstract}
Résumé
La pisciculture représente, au Cameroun, la seule alternative pour combler le déficit en poissons et réduire le niveau des importations. C'est aussi une nouvelle source de revenu pour des producteurs ruraux. Cependant, les systèmes piscicoles proposés ne sont pas assez performants sur le plan biotechnique et économique pour impulser des dynamiques marchandes. Différentes approches pour développer la pisciculture ont été appliquées, depuis les années 1990 : à une démarche agronomique de type systémique mise en œuvre dans des projets de développement est venue s'ajouter une démarche participative. Cet article propose une nouvelle approche pour contribuer au développement de la pisciculture basée sur une démarche de recherche-action en partenariat (RAP). Nous présenterons les premiers résultats obtenus sur le terrain camerounais.
\end{abstract}

Mots clés : Cameroun ; partenariat ; pisciculture ; recherche.

Thèmes : économie et développement rural ; pêche et aquaculture ; productions animales.

\section{Abstract \\ Development of small-scale commercial fish farming in Cameroon: Action research in partnership}

In Cameroon, fish farming represents the only alternative to fill in the deficit in fish and to decrease the importation levels. It is also a new source of income for the rural farmers. However, the fish farming systems that have been put forward are note efficient enough on the biotechnical and economical levels to drive commercial dynamics. Several approaches to the development of fish farming have been applied since the nineties: after a systemic agronomic approach implemented in development projects, a participatory approach emerged. This article introduces a new approach based on action research in partnership (ARP) to contribute to fish farming development. We intend to present here the first results achieved in the Cameroonian field.

Key words: Cameroon; fish culture; partnership; research.

Subjects: animal productions; economy and rural development; fishing and aquaculture.

"... la réussite de la nouvelle activité économique ainsi apportée au pays de l'Ouest Cameroun est donc en très bonne voie. La bataille n'est cependant pas encore gagnée et l'avenir ne sera vraiment assuré que lorsque des pêches sur une période suffisamment longue auront démontré aux pisciculteurs la rentabilité des opérations..." (J. Lemasson, 1953). e poisson est très largement consommé au Cameroun. Il constitue la principale protéine animale consommée par les populations et, notamment, par les couches les plus défavorisées (Tambi, 2001). La pisciculture représente la seule alternative pour combler le déficit en poissons et réduire le niveau des importations (FAO, 2007). C'est aussi une source de diversification de revenu pour des producteurs ruraux. 
Cependant, malgré une implantation de la pisciculture dès les années 1940 à l'ère coloniale, poursuivie après les indépendances essentiellement sous forme de projets de développement, on peut considérer que la pisciculture en Afrique subsaharienne, à l'orée du XxI ${ }^{\mathrm{e}}$, n'a toujours pas décollé.

Dans ce contexte, au cours des années 1990, deux options majeures pour appréhender et développer la pisciculture rurale émergent en Afrique subsaharienne (Stomal et Weigel, 1998) : un développement local basé sur une démarche agronomique de type systémique et une recherche en partenariat paysanschercheurs privilégiant une approche participative. D’une façon générale, ces démarches prennent peu en compte les aspects sociaux et culturels que les sociétés locales développent autour du poisson. Cela fera l'objet de la première partie. Nous émettons les hypothèses que :

- la recherche-action en partenariat (RAP) est plus apte à prendre en charge les différentes dimensions de l'activité piscicole (technique, économique, culturelle et sociale), facilitant ainsi l'initiation d'un processus d'innovation sociotechnique et organisationnel ;

- le système de pisciculture se construit en valorisant les avantages concurrentiels et les opportunités d'un territoire (Bureth et Llerena, 1992).

Ainsi, le présent projet de Construction de l'innovation piscicole (CIP) en partenariat prend en compte les savoirs locaux issus de la tradition des Mbô en matière de gestion des ressources en poissons et l'expérience piscicole de producteurs acquise à travers les projets de développement et leurs propres pratiques dans deux localités du département de Ménoua, Ouest Cameroun (deuxième partie).

Enfin, nous présenterons les premiers résultats obtenus sur le terrain camerounais par la RAP et nous conclurons avec un rapide bilan sur les intérêts et limites de la RAP.

\section{Approches antérieures}

\section{Inspiration systémique}

Dans les années 1990, l'Association pisciculture et développement rural en Afrique tropicale humide-France (APDRA-F) aborde la pisciculture suivant une démarche agronomique de type systémique (Oswald et Chamoin, 2000). Des systèmes de pisciculture jugés performants sont proposés en fonction des caractéristiques du système agraire et de l'abondance des facteurs de production : modèle périurbain semi-intensif en intrants versus modèle rural à faible niveau d'intrants. Ce dernier système sera historiquement développé avec un succès certain en Côte-d'Ivoire (Stomal et Weigel, 1998) et promu dans la zone forestierre humide de plusieurs pays dont la Guinée et récemment le Cameroun (province du Centre). Le transfert du système proposé est précédé par une contractualisation d'engagements réciproques individuels et collectifs entre le projet et les candidats à la pisciculture. La sélection des candidats repose principalement sur les qualités du site et la capacité du candidat à réaliser l'aménagement des étangs. La participation aux formations et la facilitation des apprentissages constituent un engagement fort des parties prenantes du projet.

Le contrat prévoit une application stricte du modèle par les pisciculteurs en contrepartie d'un engagement du projet sur les résultats technico-économiques. Cependant, cela n'exclut pas la réalisation des ajustements nécessaires du système de pisciculture au contexte local.

\section{Partenariat paysans-chercheurs...}

La recherche paysans-chercheurs développée par le WorldFish Center et ses partenaires vise à faciliter l'adoption de techniques par la réalisation simultanée d'essais contrôlés en milieu paysan et en stations de recherche (Brummett et Noble, 1995; Brummett et al., 2004). En Afrique, elle a été initialement appliquée au Malawi, au Ghana puis récemment au Cameroun, sans permettre la mise en œuvre d'une pisciculture marchande rentable. Elle utilise les outils des démarches participatives et, notamment une représentation graphique des flux de ressources biologiques au sein de l'exploitation agricole. L'évaluation participative des ressources permet d'engager un dialogue entre chercheurs et paysans sur les intégrations possibles et les technologies disponibles. La sélection de la technologie est laissée aux producteurs, le chercheur se contentant d'éviter des essais qui conduiraient à des échecs certains. Si les apprentissages mutuels sont favorisés à travers la restitution des résultats, cette approche demeure dans une logique de transfert de techniques proposées par les chercheurs.

\section{Recherche-action}

La démarche de recherche-action (Chia, 2004; Liu 1992) vise un objectif dual: trouver des solutions aux problèmes identifiés avec et pour les acteurs et produire par l'expérimentation des connaissances d'ordre local (contextuel) et génériques.

Cet objectif dual favorise les apprentissages et l'autonomie des acteurs (empowerment), notamment par l'action collective (Scoones et Thompson, 1999).

Chemin faisant, de nouveaux questionnements peuvent émerger au sein d'un cycle et donner naissance à un nouveau cycle. La notion de cycle renvoie à une succession annuelle de phases de problématisation, de formalisation des activités à travers des protocoles, d'expérimentation et de bilan.

Si nous qualifions la recherche-action aussi de partenarial (RAP), c'est pour mettre l'accent sur l'implication des acteurs non seulement dans la phase d'expérimentation ou de validation mais dans toutes les phases de la recherche, y compris sa gouvernance (Chia et al., 2008).

La démarche part du principe que la mise en place de processus de construction d'innovation se fait à travers des traductions successives, des expériences, des représentations, des questionnements des uns et des autres en un langage qui soit partagé par tous (Akrich et al., 1988). Ces traductions doivent permettre de faire converger des stratégies individuelles et collectives, et des représentations diverses du problème rencontré vers un point de passage obligé qui facilite l'engagement dans un projet commun (Callon, 1986).

Ces processus de traduction et de construction d'un langage commun permettent d'intéresser et d'enrôler dans la poursuite d'objectifs communs, des acteurs divers, chemin faisant (Akrich et al., 1988). La construction d'innovations et la reconfiguration des rapports sociaux vont de pair, d'où l'importance de regarder la construction des innovations sous un angle d'action collective. De l'intéressement, puis de l'enrôlement de différents 
Tableau 1. État de la pisciculture dans le département de la Ménoua en 2004.

Table 1. Current situation of fish farming in the Menoua Department in 2004.

\begin{tabular}{|c|c|c|c|c|c|}
\hline Arrondissement & $\begin{array}{c}\text { Nombre } \\
\text { d'exploitants agricoles }^{a}\end{array}$ & $\begin{array}{c}\text { Nombre } \\
\text { de pisciculteurs recensés }\end{array}$ & $\begin{array}{l}\text { Nombre total } \\
\text { d'étangs }\end{array}$ & $\begin{array}{c}\text { Superficie } \\
\text { moyenne }\left(\mathrm{m}^{2}\right)\end{array}$ & $\begin{array}{c}\text { Étangs } \\
\text { abandonnés (\%) }\end{array}$ \\
\hline Dschang & 9278 & 43 & 84 & 236 & 36,9 \\
\hline Nkong-Ni & 13281 & 20 & 32 & 206 & 50,0 \\
\hline Penka Michel & 17355 & 16 & 26 & 243 & 23,1 \\
\hline Fokoué & 7080 & 23 & 53 & 221 & 56,6 \\
\hline Santchou & 7356 & 31 & 268 & 44 & 64,9 \\
\hline Total & 54330 & 133 & 463 & & 55,5 \\
\hline
\end{tabular}

a Données non publiées du PNVRA.

acteurs dans la recherche dépendent la qualité et la richesse du processus de coconstruction et des apprentissages qui en découlent.

Portant sur un objet de recherche complexe, la RAP mobilise tout le long du processus des connaissances et méthodes des sciences agro-bioécologiques et socioéconomiques qui vont contribuer à construire, avec les acteurs, un réseau socio-technique piscicole.

Le réseau sociotechnique est constitué par des entités humaines (chercheurs, acteurs institutionnels, producteurs etc.) et non humaines (poissons, senne, etc.), individuelles ou collectives, définies par leurs projets et leurs identités, en interaction les uns avec les autres (Callon, 1986). Le réseau explore et exploite les spécificités physiques, économiques et sociales locales (Bureth et Llerena, 1992) pour concevoir un système de pisciculture rentable.

\section{Processus de la RAP}

\section{au Cameroun}

\section{CIP et partenariat}

Des chercheurs du Centre de coopération internationale en recherche agronomique pour le développement (Cirad), de l'Institut de recherche agricole pour le développement (Irad), de l'université de Dschang (UDs) et de l'université de Yaoundé-I (UYI) développer un projet de recherche pour étudier la possibilité de développer un système piscicole local rentable dans la province de l'Ouest du Cameroun.

\section{Du diagnostic à la formalisation de la demande sociale : la phase d'exploration}

Le projet a débuté en juin 2004 avec la réalisation d'un diagnostic sur l'insertion de la pisciculture dans les exploitations familiales agricoles du département de la Ménoua (Ouest Cameroun). Cent trentequatre exploitants ont été enquêtés lors de l'inventaire " exhaustif ", couvrant l'ensemble de la Ménoua (tableau 1), puis 17 exploitants (et leurs familles) ont fait l'objet d'entretiens approfondis à travers plusieurs visites afin de comprendre le fonctionnement global de leur exploitation (Dufumier, 1996 ; Chia, 1992).

À la suite de ces analyses-diagnostics, plusieurs restitutions-validations des résultats ont été réalisées auprès de 98 producteurs répartis en trois groupes selon leurs proximités géographiques et leur convenance dans trois localités de Ménoua : Dschang, Fokoué et Santchou. Ces réunions ont été suivies de premiers engagements réciproques. En janvier 2005, de nouvelles réu- nions de négociation ont eu lieu avec deux des trois groupes organisés en Groupement d'initiative commune (GIC). Ces négociations ont abouti à une première traduction des préoccupations des acteurs (tableau 2) en problèmes à résoudre pour chacun des deux GIC regroupant respectivement les producteurs des arrondissements de Fokoué et de Penka-Michel (collectif des pisciculteurs intensifs de Fokoué et de Penka-Michel) d'une part, et de Santchou (pêcheurs et pisciculteurs de Santchou) d'autre part.

En juin 2005, ce diagnostic a été complété par une étude socio-anthropologique de l'exploitation de la ressource poisson dans la plaine d'inondation des Mbô.

Le projet n'est donc pas impulsé par une demande des producteurs mais se construit à travers le diagnostic initial sur un dialogue entre une intention de recherche et une volonté de changement des producteurs (Chia, 2004 ; Liu, 1992). Il s'agit alors pour les partenaires de formuler un problème commun qui se décline en questions de recherche à travailler ensemble.

\section{Tableau 2. Demande sociale exprimée.}

Table 2. Social demand expressed.

\begin{tabular}{lccc}
\hline & Difficultés (\%) & Raisons d'abandon (\%) & Attentes (\%) \\
\hline Mortalités d'alevins & 22,4 & 14 & \\
Manque d'alevins & 11,2 & Non évoqué & 18,3 \\
Manque d'encadrement & 16,0 & $31^{a}$ & 19,6 \\
Manque d'argent & 21,6 & $33^{b}$ & 36,1 \\
Vente difficile & 9,6 & 13 & 1 \\
Vol & 8 & 2 & $16,9^{c}$ \\
Autres & 11,2 & 7 & \\
\hline
\end{tabular}

\footnotetext{
an incluant la mauvaise conception et réalisation des étangs.

${ }^{b}$ En incluant la non-rentabilité, la pénibilité et le manque de temps.

c Disponibilité d'aliments pour poissons.
} 
Cadre éthique

pour la coconstruction

de recherche-action commune structurante

En octobre 2005, la négociation d'un cadre éthique commun a abouti à la signature d'une convention tripartite entre les deux collectifs de producteurs organisés en GIC et le collectif de chercheurs. Dans chaque collectif, 10 à 15 personnes sont directement impliquées dans la RAP.

La convention établit des engagements réciproques (tableau 3). Elle prévoit la création d'un comité de pilotage et d'un comité scientifique, ainsi que la fréquence des rencontres entre les partenaires (tous les 15 jours) et la durée du projet (trois ans).

Le plan d'action est établi à partir de la formulation d'une question de recherche commune structurante pour chacun des GIC : à Fokoué, comment disposer d'alevins de qualité et rendre viable l'activité piscicole? À Santchou : comment valoriser la collecte d'alevins de silures issus du milieu naturel?

Ces questions générales sont ensuite transformées en questions de recherche traitables au cours d'un cycle. Leur durée tient compte des cycles de production des poissons liés aux saisons et aux stratégies des producteurs. Chacune de ces questions fait l'objet d'un protocole d'étude ou d'expérimentation négocié et validé par les parties prenantes du projet.

\section{Du partage des pratiques piscicoles à l'expérimentation}

Pour élaborer les protocoles expérimentaux du premier cycle, des séminaires ont été organisés sur les pratiques d'élevage lors des rencontres bimensuelles. À la suite de ces séminaires, diverses activités de démonstration ou de partage des savoir-faire ont été conduites. Au sein de ces protocoles, les tâches sont réparties entre les membres du GIC et le collectif des chercheurs. Outre les réunions d'échanges bimensuelles entre chercheurs et producteurs, les résultats font l'objet de plusieurs restitutions intermédiaires et débats.

À l'issu du premier cycle, il s'agit d'en faire le bilan et de renégocier un nouveau cycle. On coconstruit de nouvelles problématiques de recherche et d'action. La mise en œuvre de nouveaux protocoles est facilitée par l'enrôlement dans le dispositif d'étudiants, d'enseignants chercheurs et de chercheurs porteurs de nouvelles disciplines. On assiste ainsi, au cours du temps, à une diversification et à un approfondissement des problématiques de recherche. Cette évolution entraîne un élargissement et renforcement du collectif de recherche.

Les partenaires ont aussi été amenés à négocier de nouvelles alliances auprès d'autres acteurs du territoire: agents des services techniques, maires, projets de développement, ONG, etc. Ces nouvelles alliances doivent contribuer à anticiper le désengagement des chercheurs, prévu à la fin du troisième cycle et, éventuellement, la renégociation d'un nouveau projet.

\section{Enseignements du projet CIP}

\section{À l'origine de la légitimité des parties prenantes: le diagnostic}

Dans un partenariat naissant, les incertitudes sont multiples, tant du point de vue technique que social. La qualité du diagnostic est primordiale pour limiter ces incertitudes. En particulier, face à une intention de recherche, il est important de s'assurer qu'une véritable volonté de changement existe, que les groupes stratégiques et les enjeux pour la société ont été bien identifiés et que les acteurs aient les ressources nécessaires pour atteindre l'objectif commun.

Certaines difficultés rencontrées par le projet CIP sont, pour parties, liées à une prise en compte insuffisante des stratégies d'engagement (raisons pour lesquelles les acteurs s'engagent) individuelles comme, par exemple, la satisfaction d'ambitions d'accès au pouvoir ou les attentes du bénéfice de la rente du projet (Olivier de Sardan, 1995).

\section{Partenariat, une friction productive}

L'objectif commun est le produit des logiques différentes des parties prenantes. Ces logiques vont s'exprimer et vont se négocier à travers les pratiques, les représentations et les croyances.

\section{Tableau 3. Engagements réciproques extraits de la convention signée entre les différents collectifs.}

Table 3. Mutual agreements based on the contract signed by the different groups.

\section{Article 4 : engagement des producteurs}

Article 5 : engagement des chercheurs

4.1. Favoriser l'action collective et participer aux réunions de travail de leur GIC respectif

4.2. Mettre en commun les savoir-faire au sein de leur GIC et plus largement ceux de tous les acteurs du projet RAP

4.3. Partager avec l'ensemble des acteurs du projet RAP les informations techniques et financières, susceptibles de faire avancer le projet (ne pas " cacher " les informations)

4.4. Favoriser la mise en œuvre des protocoles élaborés ensemble et respecter les consignes arrêtées ensemble

4.5. Appliquer les nouvelles techniques élaborées en commun et les concernant

4.6. Permettre aux chercheurs d'utiliser les données dans des publications scientifiques ou de développement
5.1. Contribuer au développement des investigations décidées collectivement avec les acteurs

5.2. Mettre au service du projet toutes leurs connaissances

5.3. Restituer les résultats du projet RAP régulièrement dans un langage compréhensible

5.4. Maintenir des contacts réguliers (bimensuels) avec les GIC

5.5 Faciliter les contacts avec d'autres institutions et organismes susceptibles d'aider la réalisation du projet

5.6. Mentionner dans les publications les origines des données 


\section{Tableau 4. Les modalités de l'émergence des principales controverses de la recherche-action en partenariat au Cameroun.}

Table 4. Forms of the emergence of the main controversies of the Action Research in partnership in Cameroon.

\begin{tabular}{|c|c|c|c|c|c|}
\hline Déclencheur & Contrainte & Acteurs & Actions & Résultats & Controverse \\
\hline Diagnostic & $\begin{array}{l}\text { Pas de chercheur } \\
\text { des sciences } \\
\text { sociales }\end{array}$ & $\begin{array}{l}\text { Chercheurs (3) } \\
\text { Enquêteurs (3) } \\
\text { Producteurs }\end{array}$ & $\begin{array}{l}\text { Enquêtes } \\
\text { Entretiens } \\
\text { Restitution } \\
\text { Négociation }\end{array}$ & $\begin{array}{l}\text { Réalité } \\
\text { de la pisciculture } \\
\text { Première traduction } \\
\text { de la demande } \\
\text { en problèmes }\end{array}$ & $\begin{array}{l}\text { Pisciculture } \\
\text { non rentable } \\
\text { Déclin des pratiques } \\
\text { (plaine des Mbôs) }\end{array}$ \\
\hline Diagnostic & Langue et temps & $\begin{array}{l}\text { Anthropologues } \\
\text { et producteurs }\end{array}$ & $\begin{array}{l}\text { Enquêtes } \\
\text { dans la plaine } \\
\text { des Mbôs }\end{array}$ & $\begin{array}{l}\text { Qualification } \\
\text { des pratiques } \\
\text { et validation } \\
\text { d'une typologie } \\
\text { fondée } \\
\text { sur les pratiques }\end{array}$ & $\begin{array}{l}\text { Place des pratiques } \\
\text { héritées des pères } \\
\text { dans le changement } \\
\text { proposé }\end{array}$ \\
\hline $\begin{array}{l}\text { Négociation } \\
\text { du dispositif RAP }\end{array}$ & $\begin{array}{l}\text { Nouveauté } \\
\text { de l'approche }\end{array}$ & $\begin{array}{l}\text { Chercheurs } \\
\text { producteurs }\end{array}$ & $\begin{array}{l}\text { Réunions } \\
\text { Ateliers } \\
\text { sur les pratiques } \\
\text { Travaux pratiques }\end{array}$ & $\begin{array}{l}\text { Signature } \\
\text { d'une convention } \\
\text { Définition } \\
\text { des questions } \\
\text { structurantes }\end{array}$ & $\begin{array}{l}\text { Partenariat } \\
\text { déséquilibré } \\
\text { en faveur } \\
\text { des chercheurs: } \\
\text { pas de subvention }\end{array}$ \\
\hline $\begin{array}{l}\text { Comment produire } \\
\text { de gros poissons? }\end{array}$ & $\begin{array}{l}\text { Producteur } \\
\text { se perçoit comme } \\
\text { un exécutant }\end{array}$ & $\begin{array}{l}\text { Étudiant } \\
\text { en agronomie } \\
\text { producteurs (12) }\end{array}$ & $\begin{array}{l}\text { Élaboration } \\
\text { des règles } \\
\text { communes } \\
\text { pour pratiquer } \\
\text { la pisciculture (point }\end{array}$ & $\begin{array}{l}\text { Obtention } \\
\text { de gros poissons } \\
\text { Changement } \\
\text { des représentations }\end{array}$ & $\begin{array}{l}\text { Densité de poissons } \\
\text { à la mise en charge } \\
\text { pisciculture: } \\
\text { non marchande } \\
\text { ou marchande? }\end{array}$ \\
\hline $\begin{array}{l}\text { Comment faciliter } \\
\text { I'action collective? }\end{array}$ & $\begin{array}{l}\text { Dysfonctionnement } \\
\text { du GIC }\end{array}$ & $\begin{array}{l}\text { Chercheurs } \\
\text { et étudiante } \\
\text { en sociologie } \\
\text { producteurs (12) }\end{array}$ & de passage obligé) & $\begin{array}{l}\text { La question } \\
\text { de l'organisation } \\
\text { est posée }\end{array}$ & $\begin{array}{l}\text { Place } \\
\text { de la sociologie } \\
\text { dans la résolution } \\
\text { des problèmes }\end{array}$ \\
\hline
\end{tabular}

Le dispositif de la RAP, notamment le cadre éthique en formalisant les engagements réciproques, instaure des gardefous indispensables sans pour autant supprimer toute fiction et friction entre les parties prenantes (Soulard et al., 2007), car les incertitudes sont nombreuses et importantes tant au niveau des pratiques que de l'environnement. Il ne saurait être question de les "domestiquer" (ou les enfermer) dans un contrat. Les controverses et tensions entre les partenaires (tableau 4) sont source de redéfinition de la situation, de remise en cause du projet et peuvent donc être rendues constructives : le malentendu sur la demande de financement de la part de producteurs, par exemple, est source de tensions, et depuis le début du projet, mais il amène à poser la question essentielle de leur autonomisation. En effet, une appropriation progressive du projet et de ses objectifs amène un déplacement de la question : qu'est-ce que vous nous apportez pour la réussite du projet? à : comment pouvons-nous nous organiser pour que notre projet réussisse?
Cette friction productive accélère les changements des représentations et des pratiques de toutes les parties prenantes et favorise ainsi la production de connaissances dans et pour l'action.

\section{Pour de nouveaux savoirs : changer pratiques et représentations}

Les controverses issues du partenariat, les protocoles en action et les restitutions des résultats modifient les représentations de toutes les parties prenantes sur leurs pratiques techniques et organisationnelles, et créent ainsi des apprentissages croisés (Hatchuel, 2001).

Les deux premiers cycles de la RAP sont caractérisés par :

- la satisfaction des producteurs du GIC COPIFOPEM (Fokoué et Penka-Michel) au regard des récoltes de poissons lors de la vidange des étangs (tableau 5). Les premiers cycles ont été très riches en termes de production et de partage de connaissances pour élaborer les règles du modèle local de pisciculture. Les producteurs perçoivent la pisciculture comme une activité capable de produire de gros poissons et de générer des revenus ;

- l'engagement d'un noyau de producteurs du GIC PEPISA (Santchou) dans l'organisation de la collecte, la conservation et commercialisation d'alevins de silure issus du milieu naturel pour approvisionner la pisciculture ;

- l'émergence de nouvelles questions à traiter lors du prochain cycle portant sur l'organisation des producteurs, les pratiques liées à la consommation des poissons et les formes de coordination pour la professionnalisation.

\section{Conclusion}

Le processus de RAP présenté a pour ambition de traiter des questions com- 
Tableau 5. Caractéristiques des récoltes au cours du deuxième cycle de la RAP.

Table 5. Characteristics of harvests during the second partnership-based-Action-Research cycle.

\begin{tabular}{|c|c|c|c|c|c|c|c|}
\hline Producteur & Étang $\left(\mathrm{m}^{2}\right)$ & De (jours) & $\mathrm{Bi}(\mathbf{k g})$ & Bf (kg) & Gp (kg) & Rdt (kg/ha par an) & PMf tilapia $\bigcirc \sigma^{x}(\mathbf{g})$ \\
\hline 1 & 350 & 382 & 18,5 & 112,7 & 94,2 & 2571 & $143 \pm 17$ \\
\hline 2 & 193 & 395 & 6,6 & 83,4 & 76,8 & 3678 & $198 \pm 25$ \\
\hline 3 & 180 & 320 & 3,5 & 41,2 & 37,7 & 2389 & $182 \pm 86$ \\
\hline 4 & 108 & 389 & 7,8 & 39,6 & 31,8 & 2763 & $161 \pm 29$ \\
\hline 5 & 160 & 391 & 3,1 & 122,8 & 119,7 & 6981 & $225 \pm 44$ \\
\hline 6 & 153 & 390 & 5,9 & 50,0 & 44,1 & 2698 & $209 \pm 29$ \\
\hline 7 & 108 & 390 & 5,6 & 37,6 & 32,0 & 2770 & $220 \pm 36$ \\
\hline 8 & 150 & 387 & 5,8 & 50,0 & 44,2 & 2781 & $191 \pm 44$ \\
\hline
\end{tabular}

$\mathrm{Bi}$ : biomasse initiale ; $\mathrm{Bf}$ : biomasse finale ; Gp : gain de poids ; De : durée d'élevage ; Rdt : rendement ; PMf : poids moyen final.

plexes (Chia, 2004 ; Liu, 1992) de la pisciculture rurale comprise comme la construction d'un modèle de production localisé et d'un réseau socio-technique et organisationnel (da Silva et al., 2005). La RAP, en tant que démarche, même si elle s'est engagée dans un développement local, vise à produire, à partir de connaissances "situées ", des connaissances génériques (Liu, 1992) et à alimenter le débat sur le "décollage " de la pisciculture en Afrique subsaharienne et en particulier au Cameroun.

La RAP augmente la capacité d'action et l'autonomie des acteurs. À l'issue des deux premiers cycles de la RAP, les producteurs sont davantage capables de coconcevoir les protocoles expérimentaux avec les chercheurs et plus largement d'orienter le travail de recherche. Toutefois, une durée de deux ans n'est pas suffisant pour conclure à la supériorité de la RAP quand il s'agit de mettre en place une pisciculture marchande rentable.

La RAP exige, de la part des chercheurs, un changement de posture qui se traduit par une multitude de fonctions et/ou de capacités : traducteur, animateur, médiateur, chercheur, porte-parole, etc. Ces fonctions ne vont pas de soi et les compétences doivent être acquises rapidement et au cours de l'action. Or, la formation des chercheurs et le système d'évaluation ne favorisent pas la participation des chercheurs.

Si les résultats de la RAP sont encourageants, nous devrions porter un regard particulier à la question de l'engagement des acteurs et à la gouvernance de la RAP (comité de pilotage, etc.), et, sur le plan scientifique, à la façon de produire des connaissances actionnables légitimées afin que celles-ci deviennent des savoirs scientifiques actionnables et constituent la base d'un nouveau paradigme pour la recherche agronomique ou l'ingénierie du développement rural (Chanal et al., 1997).

\section{Remerciements}

Nous remercions le Projet de renforcement des partenariats dans la recherche agronomique au Cameroun (REPARAC), les groupes d'initiatives communes COPIFOPEM et PEPISA et tous nos autres partenaires.

\section{Références}

Akrich, M, Callon M, Latour B. À quoi tient le succès des innovations? Premier épisode: l'art de l'intéressement. Deuxième épisode: l'art de choisir les bons porte-paroles. Gérer et comprendre, 1988.

Brummett RE, Noble RP. Farmer-Scientist Research Partnerships and Smallholder Integrated Aquaculture in Malawi. In: Symoens JJ, Micha JC, eds. The Management of Integrated Freshwater Agro-Piscicultural Ecosystems in Tropical Areas Wageningen. Wageningen (Pays-Bas) ; Brussels : Technical Centre for Agricultural and Rural Cooperation ; Royal Academy of Overseas Sciences, 1995.

Brummett RE, Jamu D, Jere J, Pouomogne V. A farmer-participatory approach to aquaculture technology development and dissemination. Uganda J Agricult Sci 2004 ; 9 : 530-6.

Bureth A, Llerena P. Système local d'innovation: approche théorique et premiers résultats empiriques. In : Actes du colloque industrie et territoire: les systèmes productifs localisés, 21 et 22 octobre 1992. Grenoble: Institut de recherche économique sur la production et le développement, 1992: 369-93.
Callon M. Éléments pour une sociologie de la traduction: la domestication des coquilles Saint-Jacques et des marins-pêcheurs dans la baie de Saint-Brieuc. Année Sociol 1986 (36): 169-208.

Chanal V, Lesca H, Martinet AC. Vers une ingénierie de la recherche en sciences de gestion. Rev Fr de Gestion 1997 : 41-51.

Chia E. Une "recherche-clinique ": proposition méthodologique pour l'analyse des pratiques de trésorerie des agriculteurs (étude de cas en Lorraine). Etudes et recherches sur les systèmes agraires et le développement, $\mathrm{n} \circ 12$. Paris : Inra SAD, 1992.

Chia E. Principes, méthodes de la recherche en partenariat: une proposition pour la traction animale. Rev El Med Vet Pays Trop 2004; 57 $(3-4)$ : 233-40.

Chia E, Barlet B, Tomedi Eyango M, Pouomogne $\mathrm{V}$, Mikolasek $\mathrm{O}$. Co-construction of a local fish culture system: Case study in Western Cameroon. In: Dedieu B, ed. Empowerment of the rural actors. A renewal of farming systems perspectives. 8 th European IFSA Symposium, 6-10 July 2008, Clermond-Ferrand. Paris : Inra, 2008 (cédérom).

da Silva NJR, Beuret JE, Mikolasek O, Fontenelle G, Dabbadie L, Martins MIEG. Dynamiques de développement de la pisciculture et politiques publiques dans la vallée du Ribeira, État de São Paulo (Brésil). Cah Agric 2005 ; 14 : 59-63.

Dufumier M. Les projets de développement agricole. Paris : CTA-Karthala, 1996.

FAO. The State of World Fisheries and Aquaculture 2006. Roma: FAO Fisheries Department, 2007.

Hatchuel A. Rapports de prescription et conception collective du travail. In: Les ergonomes, les prescripteurs et les prescriptions. Collections Actes. Bordeaux : Éditions du laboratoire d'ergonomie des systèmes complexes, université Victor-Segalen, Bordeaux-II, 2001.

Lemasson J. Chronique piscicole. La pisciculture dans I'Ouest Cameroun. Rev Bois For Trop $1953 ;(30): 38$.

Liu M. Présentation de la recherche-action: présentation, déroulement et résultats. Rev Int Syst 1992 ; 6 : 293-311. 
Olivier de Sardan JP. Anthropologie et développement. Essai en socio-anthropologie du changement social. Paris: APAD-Karthala, 1995.

Oswald $\mathrm{M}$, Chamoin J. La réponse à la demande ivoirienne d'aménagement piscicole: un exemple d'outil d'aménagement d'une campagne d'Afrique forestière. In : Colloque international de l'eau, l'aménagement du territoire et le développement durable, 1011 février 2000. Paris: Académie de l'eau et l'Agence de I'eau, 2000.
Soulard CT, Compagnone C, Lémery B. La recherche en partenariat : entre fiction et friction. Natures Sciences Sociétés 2007; 15: 13-22.

Scoones I, Thompson J. La reconnaissance du savoir rural. Savoir des populations, recherche agricole et vulgarisation. Paris : Karthala, 1999.

Stomal B, Weigel JY. Mythes et réalités des aquacultures africaines. Afr Contemp 1998; (187) : 60-76.
Tambi NE. Analysis of household attitudes toward the purchase of livestock products and fish in Cameroon. Agric Econ 2001; 26: 135-47. 